\title{
Opening Note: In honor of Joseph A. Wolf on the occasion of his 80th birthday
}

\section{Claudio Gorodski ${ }^{1}$}

Published online: 27 August 2018

(c) Instituto de Matemática e Estatística da Universidade de São Paulo 2018

It is a great honor for us at the São Paulo Journal of Mathematical Sciences to publish a special issue dedicated to Joe Wolf. This collection of articles reflects the wide scope of Joe's interests and influence, with subjects ranging from representation theory and harmonic analysis to differential geometry and complex analysis.

The year 2016 marked his 80th birthday and, in addition, the 50th anniversary of his 2-month visit to the University of São Paulo. In 1966 I was only 1 year old, but I met Joe in 1989 as a second year Ph.D. student in Berkeley. There I had the privilege of attending his authoritative courses on Lie groups and symmetric spaces, and he also served on my thesis committee. After I left Berkeley, we kept in touch and met every now and then at conferences around the world.

I have always appreciated the warmth and respect he showed towards students and colleagues. So I became very enthusiastic when Slava Futorny came up with the idea of a special issue of SP Journal in honor of Joe, and even more so when I found out that Alan Huckleberry and Ivan Penkov had accepted to act as Guest Editors. Finally, I want to extend my warmest thanks to all friends of Joe who volunteered to dedicate papers to him.

Claudio Gorodski

gorodski@ime.usp.br

1 Universidade de São Paulo, São Paulo, Brazil 